\title{
What are the Alternatives of PG Plus Tests for Modified Asphalt Binders?
}

\author{
MM Tariq Morshed ${ }^{1}$, Mohammad Nazmul Hassan ${ }^{1}$, and Zahid Hossain,,* \\ ${ }^{1}$ Department of Engineering, Arkansas State University, Jonesboro, AR-72467 \\ ${ }^{2}$ Department of Civil Engineering, Arkansas State University, PO Box 1740, State University, AR-72467
}

\begin{abstract}
For characterizing the polymer modified binders, different state Departments of Transportation (DOTs) use different time consuming and empirical Performance Grade (PG) Plus test methods. Furthermore, the PG Plus tests are silent when asphalt binders are modified with chemicals such as polyphosphoric acid (PPA). But, the effects of the polymer are not accurately identified through these conventional tests such as Elastic Recovery (ER) and tenacity. The main goal of this study is to recommend alternative test method(s), which can possibly be pursued by using, a Dynamic Shear Rheometer (DSR). Thus, Multiple Stress Creep and Recovery (MSCR), ER-DSR, Frequency Sweep, and Binder Yield Energy Test (BYET) are being explored to find their effectiveness. Three PG binders (PG 64-22, PG 70-22 and PG 76-22) have been selected for this investigation. These binders have been prepared with styrene-butadienestyrene (SBS) polymer, PPA, or a combination of both. Further, chemical tests such as SARA (Saturate, Aromatic, Resin, and Asphaltene) analysis and FTIR (Fourier-transform Infrared spectroscopy) are also being explored to fulfill the objectives. Preliminary findings suggest that the PG Plus tests deem to be obsolete. Also, a single test procedure is not sufficient to identify the presence and effectiveness of modifiers in the PG binders.
\end{abstract}

\section{Introduction}

The uses of polymer modified asphalt binders have been extensively increased in pavement engineering due to high resistance against rutting, thermal cracking, fatigue cracking, stripping, and temperature susceptibility $[1,2]$. But the existing Performance Grade (PG) test methods are not suitable to determine the mechanical properties of the polymer modified binders beyond the linear viscoelastic range. The conventional Superpave tests were developed only to characterize the unmodified asphalt binders [3]. Therefore, many state Departments of Transportation (DOTs) including Arkansas Department of Transportation (ArDOT) use PG Plus tests such as Elastic Recovery (ER) [4] to characterize the polymer modified binders to examine the existence of polymer. But, there is no uniform guideline to conduct the ER test and analyze the test results; each state has its own ER sample preparation procedures, test temperature, and data analysis protocols [5]. Moreover, with the invent of chemical modifiers such as polyphosphoric acid (PPA), manufacturer occasionally markets chemically-modified high-grade PG binders. However, the Plus tests are silent in the cases of chemicallymodified PG binders.

For characterizing PG asphalt binders, many state agencies follow AASHTO M320 [6] "Standard Specification for Performance-Graded Asphalt Binder." However, the applicability of AASHTO M320 [6] specification for polymer-modified asphalt binders has been questioned by many industries and state highway agencies. AASHTO M332 [7] "Performance-Graded Asphalt Binder Using the Multiple-Stress CreepRecovery (MSCR) Test" has been suggested as a substitution of AASHTO M320 [6]. It is proposed for a better understanding of the polymer effect inside the modified binder [8]. The MSCR parameters i.e. percent recovery $(\% \mathrm{R})$ and non-recoverable creep compliance (Jnr) have a better relationship with the permanent deformation [9]. On the other hand, new test methods such as AASHTO TP 101 [10] "Standard method of test for estimating damage tolerance of asphalt binders using the linear amplitude sweep" has been developed by professionals. AASHTO TP 101 [10] is often called as the Linear Amplitude Sweep (LAS) test method. The LAS test method is used to predict the fatigue life of the asphalt binders $[11,12]$. In the meantime, AASHTO TP 123 [13] "Measuring asphalt binder yield energy and elastic recovery using the dynamic shear rheometer" has also been developed. This test was a combination of the binder yield energy test (BYET) and DSR-based elastic recovery (DSR-ER) test [14]. In this test method, a monotonic constant shear load is applied on the asphalt binder at an intermediate temperature. It is claimed that AASHTO TP 123 [13] can promisingly predict lowtemperature fatigue and thermal cracking [15].

Further, it is found that some refineries occasionally manufacture high-grade asphalt binders through chemical modifications. Among the chemicals, PPA is predominately being used. However, the PG Plus tests or any of the aforementioned mechanical or rheological tests can be used to evaluate their performance properties

\footnotetext{
* Corresponding author: mhossain $@$ astate.edu
} 
related to rutting and fatigue damages. Mechanical tests may not be appropriate to evaluate chemically modified binders, but chemical tests such as SARA (saturate, aromatic, resin and asphaltene) analysis and FTIR (Fourier-transform Infrared spectroscopy) may provide some insights about them. The FTIR has been reported as the first logical step in identifying a polymer into the modified asphalt binder. The FTIR test is also used for quality control of materials and for contamination analysis. However, the FTIR peak(s) to identify PPA and some other chemicals in the binder have not been identified yet. Thus, research is needed to find the appropriate test method (s) to characterize polymer and chemical modified binders.

\section{Objectives}

The main objective of this study is to recommend effective test method (s) to evaluate modified asphalt binders as replacements of the ER test. Specific objectives are to: (i) perform rheological tests ArDOT certified binders according to MSCR, LAS, and ER-DSR test methods, (ii) conduct chemical tests such as SARA and FTIR of the binders and establish their relationship with mechanical test results; (iii) develop guidelines toward adopting the proposed test method so that neither suppliers nor users are penalized.

\section{Materials and methodologies}

\subsection{Materials}

Three ARDOT-certified asphalt binders (PG 64-22, PG 70-22 and PG 76-22) were selected for laboratory testing. In particular, unmodified PG 64-22, PPAmodified PG 70-22, SBS-modified PG 70-22, and SBS+PPA modified PG 76-22 from two different sources have been collected. The nomenclature was developed for the study to identify the samples and shown in Table 1.

Table 1. Nomenclature.

\begin{tabular}{|c|c|c|c|}
\hline \multirow[b]{2}{*}{$\begin{array}{c}\text { Performance } \\
\text { Grade }\end{array}$} & \multirow[b]{2}{*}{$\begin{array}{l}\text { Name of } \\
\text { Modifier }\end{array}$} & \multicolumn{2}{|c|}{ Name of the Binder } \\
\hline & & $\begin{array}{c}\text { Arabian } \\
\text { Crude } \\
\text { Source (S1) }\end{array}$ & $\begin{array}{c}\text { Canadian } \\
\text { Crude } \\
\text { Source (S2) }\end{array}$ \\
\hline PG 64-22 & N/A & S1B1 & S2B1 \\
\hline PG 70-22 & PPA & S1B2 & S2B2 \\
\hline PG 70-22 & SBS & S1B3 & S2B3 \\
\hline PG 76-22 & SBS+PPA & S1B4 & S2B4 \\
\hline
\end{tabular}

\subsection{Experiments}

Routine Superpave tests such as Rotational Viscometer (RV) (AASHTO T 316), Dynamic Shear Rheometer (DSR) (AASHTO T 315), Rotational Thin-Film Oven (RTFO) (AASHTO T 240), Pressure-Aging Vessel (PAV) (AASHTO R 28) and Bending Beam Rheometer (BBR) (AASHTO T 313) are included in the test plant. The repeatability of major test results by conducting replicate samples for each test condition. At least three replicates have been tested and the average test result is presented in this manuscript.

The Elastic Recovery test data for these binders have been collected The MSCR test is being conducted according to AASHTO T 350 using a DSR. In this method, the asphalt binder sample is tested for 10 cycles at different stress levels (i.e., $0.1 \mathrm{kPa}, 3.2 \mathrm{kPa}$, and 10 $\mathrm{kPa}$ ); each cycle consists of a constant creep stress for 1second duration followed by a 9 seconds recovery of zero stress (rest period). The performance indicators of the MSCR test are percent recovery $(\% \mathrm{R})$ and nonrecoverable creep compliance $\left(\mathrm{J}_{\mathrm{nr}}\right)$. The ER-DSR test was conducted according to AASHTO TP 123-16 [13]. Short term or long-term aged samples were used for testing. A constant strain rate of $0.0231 / \mathrm{s}$ was used to achieve $277.78 \%$ strain at $25^{\circ} \mathrm{C}$. Then the zero-shear stress was maintained to recover the sample for 30 minutes. The LAS test is conducted in accordance with AASHTO TP101 [10]. This test consists of two steps; at first, a frequency sweep test is performed to define the undamaged material response and then a linear oscillatory strain sweep is applied with strain amplitudes ranging from $0.1 \%$ to $30 \%$.

The SARA analysis is being conducted for determining the percentages of four major chemical families (Saturate, Aromatic, Resin, and Asphaltene) in the tested asphalt binders. This test is conducted according to ASTM D 4142-09. The test will be conducted on the base and modified asphalt binder samples to observe any changes in the chemical composition. The FTIR test will be conducted in accordance with ASTM E168 and ASTM E1252.

\section{RESULTS AND DISCUSSIONS}

\subsection{Rotational viscosity and shear modulus}

From the rotational viscosity test results, SBS+PPA modified binders S1B4 and S2B4 showed the highest viscosity compared to the other binders. In the case of PPA modified asphalt binders S1B2 and S2B2, at $135^{\circ} \mathrm{C}$, the viscosity values increased by $45 \%$ and $44 \%$, respectively, compared to the neat binders. On the other hand, the SBS modified binders S1B3 and S2B3 showed $73 \%$ and $97 \%$ higher viscosity compared to the binders modified with only PPA. Therefore, the mixing and compaction temperatures are expected to increase for the modified binders. RV test results are shown in Table 2 .

Table 2. Rotational viscosity (mPas.s) test results of binder samples.

\begin{tabular}{|c|c|c|c|c|c|c|c|c|}
\hline \multirow{2}{*}{ Temp. } & \multicolumn{7}{|c|}{ Binder Sample } \\
\cline { 2 - 10 } & S1B1 & S1B2 & S1B3 & S1B4 & S2B1 & S2B2 & S2B3 & S2B4 \\
\hline $135^{\circ} \mathrm{C}$ & 504 & 733 & 1,271 & 1,929 & 445 & 645 & 1,271 & 1,767 \\
\hline $150^{\circ} \mathrm{C}$ & 254 & 325 & 595 & 870 & 208 & 295 & 554 & 758 \\
\hline $165^{\circ} \mathrm{C}$ & 145 & 162 & 312 & 450 & 112 & 145 & 279 & 350 \\
\hline $180^{\circ} \mathrm{C}$ & 75 & 75 & 175 & 262 & 62 & 75 & 162 & 187 \\
\hline
\end{tabular}


The dynamic shear modulus test was conducted for unaged binders. The complex shear modulus increased, and phase angle decreased for modified asphalt binders. Therefore, the rutting factor $\left(\mathrm{G}^{*} / \sin \delta\right)$ increased for modified binders, as shown in Figure 1. The failing temperature was also increased for modified asphalt binders.

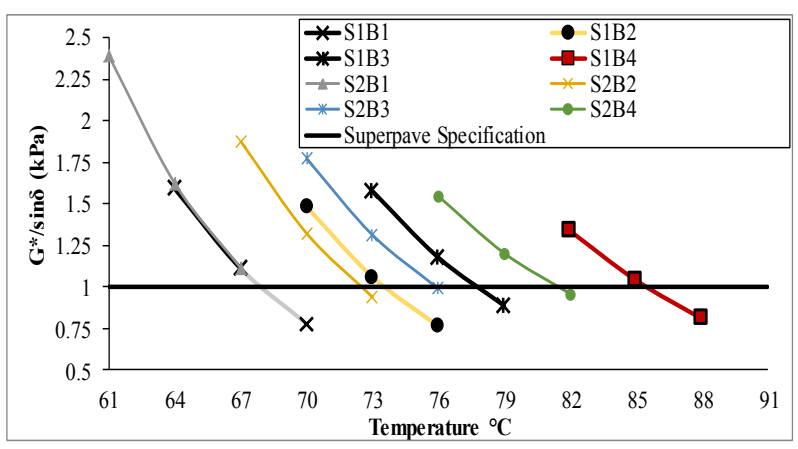

Fig. 1. $\mathrm{G}^{*} / \sin \delta$ vs temperature $\left({ }^{\circ} \mathrm{C}\right)$ curve for unaged $\mathrm{S} 1$ and $\mathrm{S} 2$ asphalt binders.

\subsection{Multiple stress creep and recovery (MSCR)}

The percent recovery was always higher for S2B4 binder as well as the non-recoverable creep compliance $\left(\mathrm{J}_{\mathrm{nr}}\right)$ was lower for the same binder. However, there was found variation in results between the same PG binders from two different sources (S1B4 and S2B4). The other results from two different sources were quite similar. The overall results are shown in Figures 2. It is suspected that at the high stress $(10 \mathrm{kPa})$ level the polymer chains are released, and the $\mathrm{J}_{\mathrm{nr}}$ values suddenly increased for S1B2 and S2B2 binders.

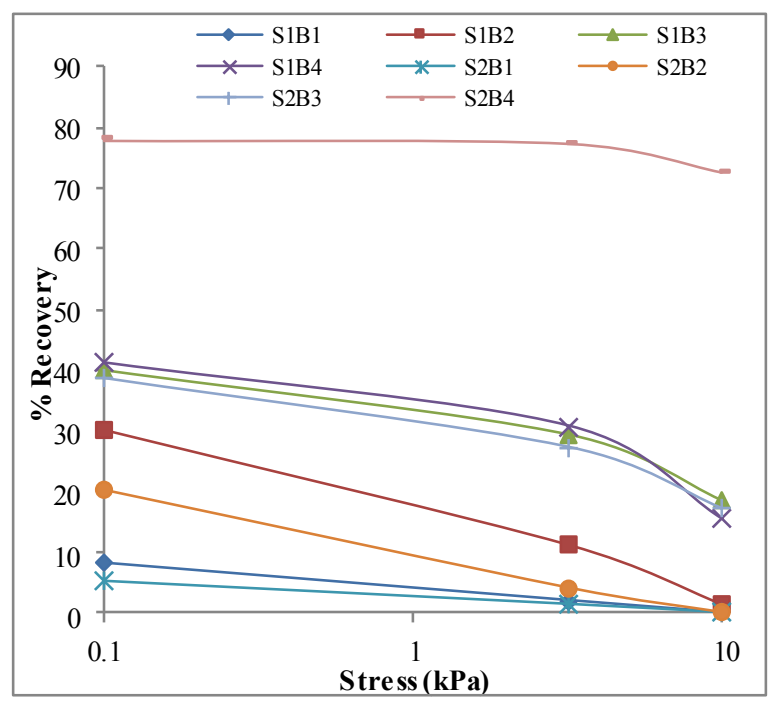

(a)

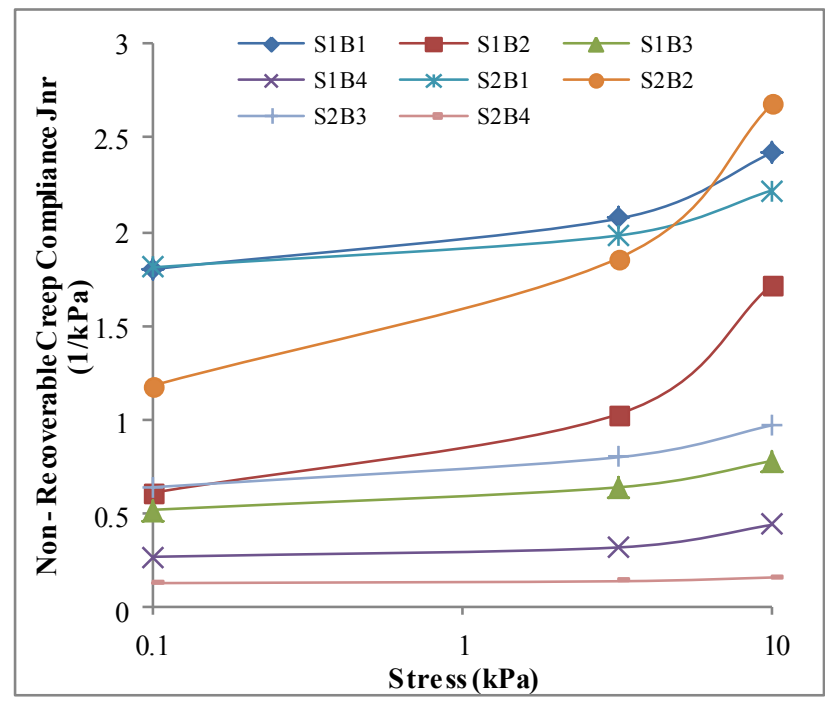

(b)

Fig. 2. MSCR test results for S1 and S2 binders: (a) percent recovery vs. stress, and (b) $\mathrm{J}_{\mathrm{nr}}$ vs stress.

\subsection{Elastic recovery}

The ER values of S1B3 and S1B4 binders are shown in Figure 3, and it is seen that the ER values are always higher than the MSCR percent recovery. The ArDOT recommends that the minimum ER values for PG 70-22 and PG 76-22 are $40 \%$ and $50 \%$, respectively.

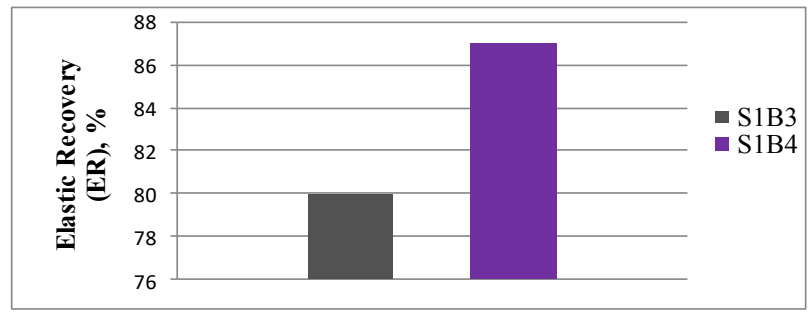

Fig. 3. Elastic recovery in percent.

\subsection{SARA analysis}

From Figure 4, unlike Source 1 binder samples, a gradual increase of saturates and asphaltenes can be detected for Source 2 binders. Moreover, it was evident that the Resin components were higher for Source 1 than Source 2 binders, whereas Source 2 binders had more aromatic components than Source 1 binders. A higher Asphaltene content (e.g., S1B2, S1B3, or S1B4) in a binder indicates a stiffer binder. As expected, S1B4, which is an SBS and PPA modified PG 76-22 binder, has the highest Asphaltene content among all binders from S1. The viscosity and DSR test results explained earlier agree with the Asphaltene contents of these binders. 


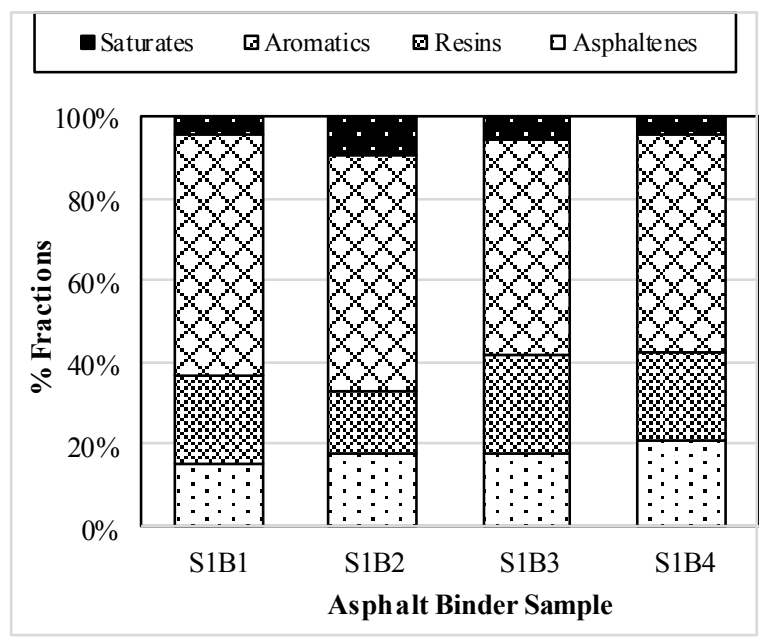

(a)

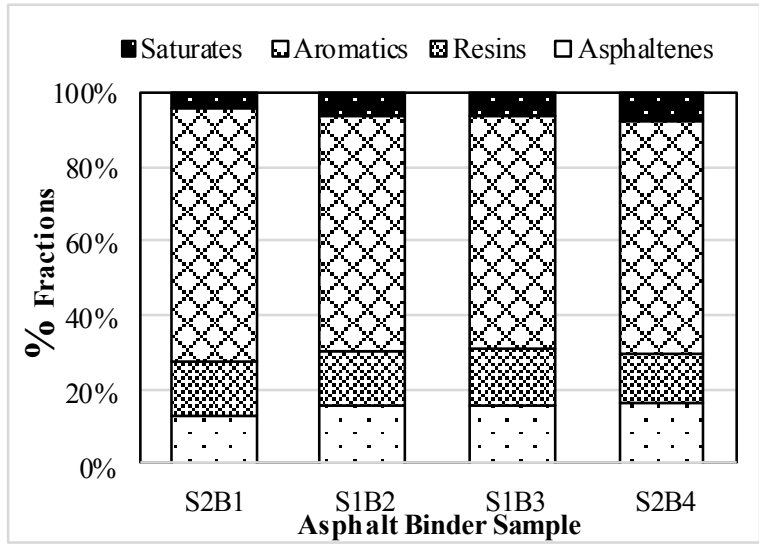

(b)

Fig. 4. SARA Fractions of the asphalt binders: (a) from S1, and (b) from $\mathrm{S} 2$.

Asphaltenes content from SARA analysis and viscosity values at $135^{\circ} \mathrm{C}$ are plotted and shown in Figure 5. An exponential relationship was found between the asphaltenes content and viscosity.

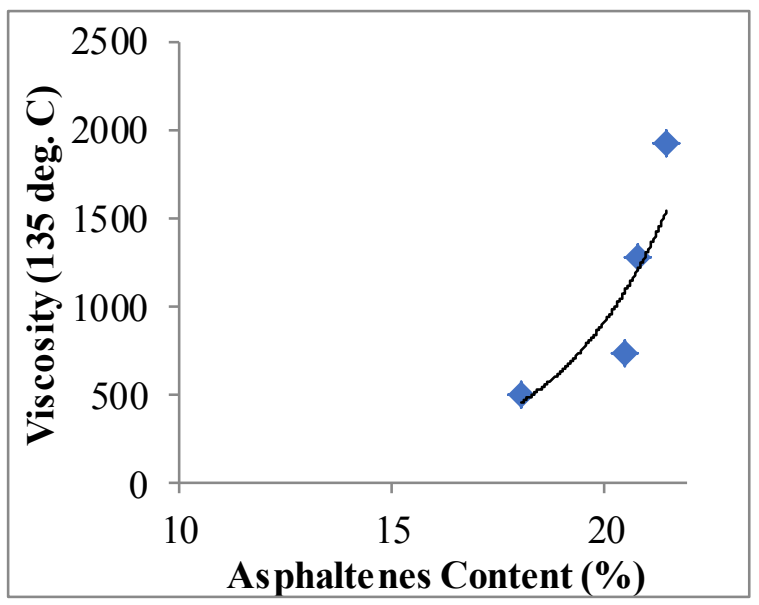

(a)

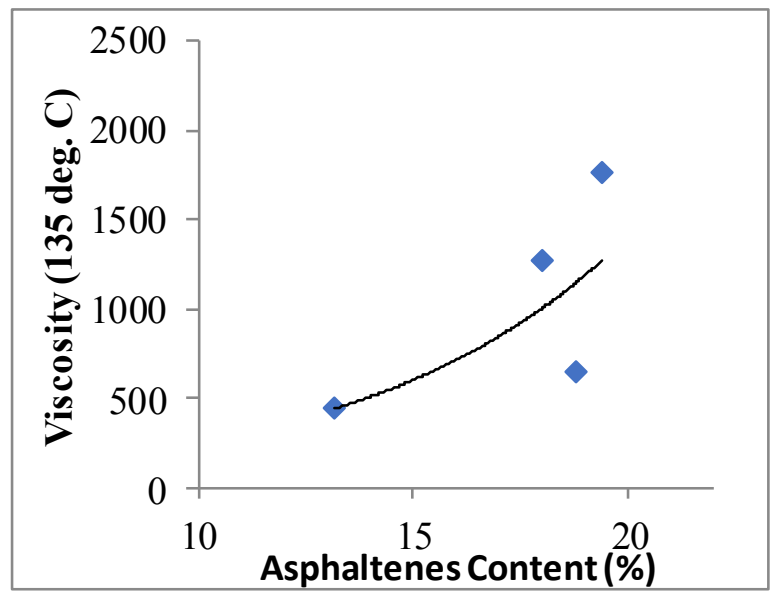

(b)

Fig. 5. Viscosity (mPa.s) vs Asphaltenes content for modified asphalt binders: (a) from S1, and (b) from S2.

\subsection{Fourier-transform infrared spectroscopy (FTIR)}

Figure 6 shows that the absorbance values for both binders follow a similar trend along with the wavenumber. The reason could be the binders have not been modified yet. Though some variation can be observed in height of the peaks between two binder samples, it is due to the variation in the chemical compositions of the neat binders from two different sources.

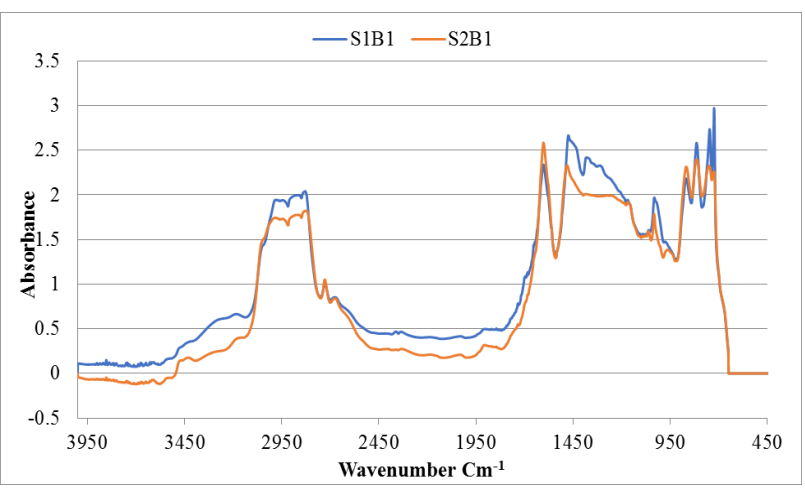

Fig. 6. FTIR test results for neat binders.

\section{Conclusions}

Commonly used unmodified and modified binders of Arkansas were tested by following different test methods. The MSCR test results provide the percent recovery and non-recoverable creep compliance, which can be used to grade the modified binders. It can also be used to calculate the stress sensitivity of the binders. This project is under progress. Beside routine rheological tests, chemical tests such as SARA and FTIR analyses are being done. The SARA analysis test results help to understand chemical fractions in the binder. The variation of the height of the peaks during the FTIR test can be used to explain the variation of chemical compositions between the neat binder samples. Based on the limited findings of this study, it is apparent that the 
ER test method lacks mechanical and scientific rigors. The MSCR test method is found to be a good alternative to the ER method, but it is not effective for the chemically modified binder. Also, not a single test method is appropriate for all types of the binder. Thus, additional research being pursued by the research team is expected to help develop meaningful correlations between the chemical test results and mechanical properties and answer the research question.

The authors gratefully acknowledge the financial support of Transportation Consortium of South-Central States to conduct this study. The authors are also thankful to Paragon Technical Services Inc. and other suppliers for providing test materials and technical support for this study.

\section{References}

1. Becker, Y., Mendez, M.P., and Rodriguez, Y. (2001). A polymer modified asphalt. Vision Technol. 9:3950.

2. Yildirim, Y. (2007). Polymer modified asphalt binders. Constr. Build. Mater. 21:66-72.

3. Clopotela, C.S., and Bahia, H.U. (2012). Importance of Elastic Recovery in the DSR for Binders and Mastics. Engineering Journal, 16(4):99-106.

4. AASHTO T 301-13. (2013). Standard Method of Test for Elastic Recovery Test of Asphalt Materials by Means of a Ductilometer. Standard Specifications for Transportation Materials and Methods of Sampling and Testing, Part 2B, 33th Edition, Washington, DC.

5. Rahaman, M.Z., Hossain, Z., and Zaman, M. (2018). Nonrecoverable Compliance and Recovery Behavior of Polymer-Modified and Reclaimed Asphalt Pavement-Modified Binders in Arkansas. Journal of Testing and Evaluation. 46(6):2483-2497.

6. AASHTO M320-16. (2016). Standard Method of Test for Performance-Graded Asphalt Binder. Standard Specifications for Transportation Materials and Methods of Sampling and Testing, Part 2B, 36th Edition, Washington, DC.

7. AASHTO M332-14. (2014). Standard Specification for Performance-Graded Asphalt Binder Using Multiple Stress Creep Recovery (MSCR) Test. Standard Specifications for Transportation Materials and Methods of Sampling and Testing, Part 2B, 34th Edition, Washington, DC.

8. Moraes, R., Swiertz, D., and Bahia, H.U. (2017). Comparison of New Test Methods and New Specifications for Rutting Resistance and Elasticity of Modified Binders. Canadian Technical Asphalt Association.

9. D’Angelo, J. (2010). New High-Temperature Binder Specification Using Multistress Creep and Recovery, Transportation Research Circular EC-147, Transportation Research Board, National Research Council, National Academies, Washington, D.C., 113.

10. AASHTO TP 101. (2014). Standard method of test for estimating damage tolerance of asphalt binders using the linear amplitude sweep. Washington D.C.
11.Johnson, C.M. (2010). Estimating asphalt binder fatigue resistance using an accelerated test method. $\mathrm{Ph} . \mathrm{D}$. dissertation, University of Wisconsin-Madison, Madison, WI.

12. Hintz, C. and Bahia, H. (2013). Simplification of linear amplitude sweep test and specification parameter. Transp. Res. Record: J. Transp. Res. Board 2370:10-16.

13. American Association of State Highway and Transportation Officials (AASHTO) TP 123. (2016). Measuring asphalt binder yield energy and elastic recovery using the dynamic shear rheometer. Washington D.C.

14. Johnson, C.M., Wen, H., and Bahia, H. (2009). Practical application of viscoelastic continuum damage theory to asphalt binder fatigue characterization, J. Asphalt Paving Technol. 78:597638.

15. Wen, H. and Bhusal, S. (2013). Toward development of a new thermal cracking test using the dynamic shear rheometer, J. Test. Eval. 41(3):1-8. 